\title{
Successful Use of the 308-nm Excimer Laser in Early Patch Stage Mycosis Fungoides
}

\author{
Andrew M. Armenta ${ }^{1}$, Krystal M. Jones ${ }^{2 *}$, Jason S. Reichenberg ${ }^{2}$ \\ ${ }^{1}$ University of Texas Medical Branch, School of Medicine, Galveston, Texas, USA \\ ${ }^{2}$ Division of Dermatology, Dell Medical School, University of Texas at Austin, Austin, TX, USA
}

\author{
*Correspondence to \\ Krystal M. Jones, MD; \\ 1400 N I-35, Department of \\ Dermatology, Clinical Education \\ Center, Suite C2.470, Austin, TX \\ 78701 (281-451-3686) \\ Email: \\ Krystalmjones2@gmail.com
}

Published online July 6, 2019

\begin{abstract}
Introduction: Mycosis fungoides (MF), a type of cutaneous T-cell lymphoma, is a rare condition with a variety of treatment options. A frequently utilized method in the treatment of early-stage MF is phototherapy. Full body phototherapy can be associated with photoaging of the skin and an increased risk of developing skin cancer. Targeted phototherapy, with a 308-nm excimer laser, may be just as effective and of a lower risk. This makes it especially useful in the treatment of patients with dysplastic nevi (DN) or other conditions which can put them at a higher risk of developing skin cancer. There are currently limited data on the treatment of early-stage MF with an excimer laser.

Case Report: This study reports on a 43-year-old female patient presented to the clinic with early-stage (la) MF. Given her history of DN, she wished to pursue targeted phototherapy with an excimer laser. Localized light treatment was performed via a 308-nm excimer laser, 3 times weekly, for a total of 17 treatments to affected lesions. Following excimer laser treatment, she had a clinical resolution of her patches. On the follow-up clinic visits, she maintained her excellent response 12 months out.

Conclusion: Targeted phototherapy with a 308-nm excimer laser may be a safer and equally effective alternative to generalized phototherapy in the treatment of early-stage MF. This case report demonstrates its efficacy and advantages over traditional generalized phototherapy.

Keywords: Mycosis fungoides; Lasers; Excimer.
\end{abstract}

\section{Introduction}

Mycosis fungoides (MF) is a rare condition with an incidence of only six cases per million annually but is the most common type of cutaneous T-cell lymphoma. ${ }^{1-3}$ Although MF can be present in younger patients, it has a peak incidence between the ages of 55 and 60 and is twice as common in males. ${ }^{2,45}$ The pathogenesis of MF is currently unclear. While environmental exposures and infectious causes of MF have been hypothesized, none have been confirmed. MF primarily develops in the skin but can metastasize to blood, lymph nodes, and visceral organs. Affected patients can present with a variety of widespread pruritic cutaneous lesions usually involving a truncal distribution although any body surface can be affected. ${ }^{6,7}$ Lesions can range from plaques and patches to areas of alopecia. They can mimic many dermatologic conditions such as eczema, psoriasis, photodermatitis, or drug reactions. Both its clinical mimicry and rarity make it a challenging diagnosis and one that can be established only after obtaining multiple cutaneous biopsies. ${ }^{8}$

Treatment guidelines for MF vary based on its staging. While more advanced stages of the disease may require systemic therapy, skin-directed treatments are useful in early stages. ${ }^{9}$ Currently, there are limitations and side effects attributed to many of the skin-directed therapies. Topical medications can cause hypersensitivity reactions, hypopigmentation, skin atrophy, irritant dermatitis, pruritus, pain and even hematologic toxicities. ${ }^{10,11}$ Other available options include radiation therapies which expose the patient to toxicities and widespread phototherapy, placing patients at risk for developing secondary cutaneous carcinomas and melanomas. ${ }^{12,13}$ Data to support targeted phototherapies are limited to case reports and case series. Excimer lasers, which are composed of noble gases and a reactive halogen gas (fluorine or chlorine), operate in the ultraviolet (UV) range between 193 and $351 \mathrm{~nm} .{ }^{14}$ They are used by clinicians for targeted phototherapy. ${ }^{14}$ They aid in the treatment of psoriasis, vitiligo, atopic dermatitis, and alopecia areata; however, there have been only a few reports in the treatment of MF with an excimer laser. ${ }^{15-17}$

\section{Case Presentation}

A 43-year-old female with a past medical history of dysplastic nevi $(\mathrm{DN})$ and a remote history of early-stage 


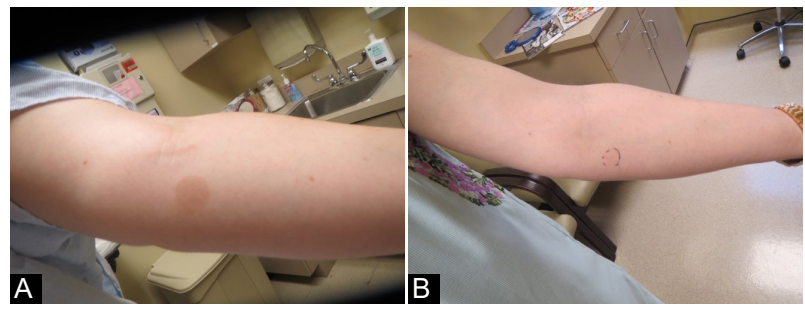

Figure 1. Clinical Presentation. (A) Tan nummular patches with fine overlying scale noted on the left volar arm. (B) The resolution of the previous patch on the left volar arm, inked for localization.

MF presented to the dermatology clinic with slightly hyperpigmented patches with a fine scale on her left volar forearm, upper left buttock and left posterior thigh. Given her history of MF and findings on physical exam, MF was suspected. Her MF had been successfully treated with narrowband UVB (NB-UVB) 5 years ago. Skin biopsy of affected areas confirmed recurrence of her MF, and following workup with a clinical lymph node exam, complete blood counts and a chemistry panel, she was diagnosed with stage 1a MF. She was given and tried using a class III topical corticosteroid for 2 months but it was discontinued due to irritation and erythema. Alternative therapies, namely phototherapy, were considered. However, given her history of DN and increased risk of melanoma, the patient preferred to use localized light treatment rather than NB-UVB. Localized light treatment was performed via a 308-nm excimer laser 3 times weekly for a total of 17 treatments to her left medial arm, left thigh and left calf. Following each tolerated treatment, her dose was gradually increased to $1288 \mathrm{~mJ} / \mathrm{cm}^{2}$. After clinical resolution of her patches, she was monitored on the follow-up visits and maintained her excellent response for 12 months.

\section{Discussion}

Historically, topical treatments have been the mainstay of early-stage (Ia-IIa) MF. More recently, with technological advances, phototherapies have become a viable option as well. Although both of these therapies have been broadly studied and proven effective, they do not come without side effects. ${ }^{10-13}$ In patients who cannot tolerate the side effects of these therapies, or have contraindications to them, alternative options need to be explored.

Traditionally, phototherapies with UVB and PUVA have been effective, but because they target both pathologic and normal skin, they can increase the risk of squamoproliferative skin neoplasms, basal cell carcinomas, or melanoma. ${ }^{12,13}$ It is recommended that maintenance phototherapy should not exceed oneyear post remission of MF in order to reduce cutaneous carcinogenesis. ${ }^{12,13}$ Patients on long term phototherapy are the most likely to develop skin cancer. ${ }^{18}$ Unfortunately, because maintenance therapy duration is limited, the majority of patients will relapse. This scenario requires further phototherapy which again can lead to carcinogenesis. One useful way to maximize benefit while minimizing risk is the usage of targeted phototherapy.

Targeted phototherapy with the 308-nm excimer laser can successfully treat early-stage MF while minimizing exposure of normal skin to the carcinogenic UV radiation. This is especially useful when dealing with a patient population who has an increased risk of skin cancer such as DN or a long history of sun exposure. An excimer laser requires fewer treatments and results in a less toxic and cumulative UV dose to healthy skin in comparison to generalized phototherapy. It can effectively deliver a higher amount of radiation in a shorter period of time to only the affected lesions, minimizing treatment duration and reducing unnecessary UV exposure and adverse effects. ${ }^{19,20}$ It may also be useful in treating refractory skin lesions and difficult to reach anatomical locations. ${ }^{20,21}$ Accepted treatment frequency is two to three treatment sessions per week, for a total of 10 to 20 treatments, or until the lesion is cleared. ${ }^{21}$ This dosing regimen is used at our institution and has resulted in clinical resolution of early-stage MF on multiple occasions. We believe when clinically appropriate, this may be both superior and less damaging to skin than currently utilized therapies.

\section{Conclusion}

There are only a limited number of cases that study the effectiveness of the 308-nm excimer laser in the treatment of early-stage MF. ${ }^{15-17}$ Generalized phototherapy has been well researched and studied, but the efficacy of targeted phototherapy with the excimer laser has not been well established. As more research is done, it may prove to be superior to traditional generalized phototherapy. It has the potential to effectively treat MF in a more directed fashion while minimizing the potential for carcinogenesis. The excimer laser may be especially useful in the treatment of MF in patients who have a high risk for cutaneous malignancy, as seen in our patient.

\section{Ethical Considerations}

Informed consent was obtained from the patient prior to any treatment procedures.

\section{Conflict of Interests}

The authors declare no conflict of interest.

\section{References}

1. Criscione VD, Weinstock MA. Incidence of cutaneous T-cell lymphoma in the United States, 1973-2002. Arch Dermatol. 2007;143(7):854-859. doi:10.1001/archderm.143.7.854

2. Sant M,AllemaniC, TereanuC,etal.Incidenceofhematologic malignancies in Europe by morphologic subtype: results of the HAEMACARE project. Blood. 2010;116(19):37243734. doi:10.1182/blood-2010-05-282632

3. Korgavkar $\mathrm{K}$, Xiong $\mathrm{M}$, Weinstock $\mathrm{M}$. Changing incidence trends of cutaneous T-cell lymphoma. JAMA Dermatol. 2013;149(11):1295-1299. doi:10.1001/ 
jamadermatol.2013.5526

4. Bradford PT, Devesa SS, Anderson WF, Toro JR. Cutaneous lymphoma incidence patterns in the United States: a population-based study of 3884 cases. Blood. 2009;113(21):5064-5073. doi:10.1182/ blood-2008-10-184168

5. Fujita A, Hamada T, Iwatsuki K. Retrospective analysis of 133 patients with cutaneous lymphomas from a single Japanese medical center between 1995 and 2008. J Dermatol. 2011;38(6):524-530. doi:10.1111/j.1346-8138.2010.01049.x

6. Demierre MF, Gan S, Jones J, Miller DR. Significant impact of cutaneous T-cell lymphoma on patients' quality of life: results of a 2005 National Cutaneous Lymphoma Foundation Survey. Cancer. 2006;107(10):2504-2511. doi:10.1002/cncr.22252

7. Pimpinelli N, Olsen EA, Santucci M, et al. Defining early mycosis fungoides. J Am Acad Dermatol. 2005;53(6):10531063. doi:10.1016/j.jaad.2005.08.057

8. Fung MA, Murphy MJ, Hoss DM, Grant-Kels JM. Practical evaluation and management of cutaneous lymphoma. J Am Acad Dermatol. 2002;46(3):325-357.

9. Shipman AR, Scarisbrick J. New Treatment Options for Mycosis Fungoides. Indian J Dermatol. 2016;61(1):119. doi:10.4103/0019-5154.174085

10. Ramsay DL, Meller JA, Zackheim HS. Topical treatment of early cutaneous T-cell lymphoma. Hematol Oncol Clin North Am. 1995;9(5):1031-1056.

11. Heald P, Mehlmauer M, Martin AG, Crowley CA, Yocum RC, Reich SD. Topical bexarotene therapy for patients with refractory or persistent early-stage cutaneous T-cell lymphoma: results of the phase III clinical trial. J Am Acad Dermatol. 2003;49(5):801-815. doi:10.1067/s0190

12. Stern RS, Nichols KT, Vakeva LH. Malignant melanoma in patients treated for psoriasis with methoxsalen (psoralen) and ultraviolet A radiation (PUVA). The PUVA Follow-Up Study. N Engl J Med. 1997;336(15):1041-1045. doi:10.1056/ nejm199704103361501

13. Stern RS. The risk of squamous cell and basal cell cancer associated with psoralen and ultraviolet A therapy: a 30-year prospective study. J Am Acad Dermatol. 2012;66(4):553562. doi:10.1016/j.jaad.2011.04.004
14. Hamzavi I, Lui H. Using light in dermatology: an update on lasers, ultraviolet phototherapy, and photodynamic therapy. Dermatol Clin. 2005;23(2):199-207. doi:10.1016/j. det.2004.11.001

15. Deaver D, Cauthen A, Cohen G, Sokol L, Glass F. Excimer laser in the treatment of mycosis fungoides. $J$ Am Acad Dermatol. 2014;70(6):1058-1060. doi:10.1016/j. jaad.2014.01.915

16. Jin SP, Jeon YK, Cho KH, Chung JH. Excimer laser therapy $(308 \mathrm{~nm})$ for mycosis fungoides palmaris et plantaris: a skin-directed and anatomically feasible treatment. $\mathrm{Br}$ J Dermatol. 2010;163(3):651-653. doi:10.1111/j.13652133.2010.09793.x

17. Nistico SP, Saraceno R, Schipani C, Costanzo A, Chimenti S. Different applications of monochromatic excimer light in skin diseases. Photomed Laser Surg. 2009;27(4):647-654. doi:10.1089/pho.2008.2317

18. Abel EA, Sendagorta E, Hoppe RT. Cutaneous malignancies and metastatic squamous cell carcinoma following topical therapies for mycosis fungoides. J Am Acad Dermatol. 1986;14(6):1029-1038.

19. Lapolla W, Yentzer BA, Bagel J, Halvorson CR, Feldman SR. A review of phototherapy protocols for psoriasis treatment. J Am Acad Dermatol. 2011;64(5):936-949. doi:10.1016/j. jaad.2009.12.054

20. Fikrle T, Pizinger K. [The use of the $308 \mathrm{~nm}$ excimer laser for the treatment of psoriasis]. J Dtsch Dermatol Ges. 2003;1(7):559-563.

21. Menter A, Korman NJ, Elmets CA, et al. Guidelines of care for the management of psoriasis and psoriatic arthritis: Section 5. Guidelines of care for the treatment of psoriasis with phototherapy and photochemotherapy. J Am Acad Dermatol. 2010;62(1):114-135. doi:10.1016/j. jaad.2009.08.026

22. Buckland M, Smith JS, Hill D, Feldman SR. A Review of Localized Office and Home Devices in the Treatment of Psoriasis. J Psoriasis Psoriatic Arthritis. 2016;2(1):22-30. doi:10.1177/247553031600200104

23. Mudigonda T, Dabade TS, Feldman SR. A review of protocols for $308 \mathrm{~nm}$ excimer laser phototherapy in psoriasis. J Drugs Dermatol. 2012;11(1):92-97. 